\title{
Mariner's Performances and the Behavior Fluctuation Affecting Navigational Safety
}

\author{
Tae-Goun Kim*, Hiroaki Kobayashi**, † Chae-Uk Song \\ * Division of Maritime Transportation Science, Korea Maritime University, Busan 606-791, Republic. of Korea \\ ** Department of Applied Marine Environmental Studies, Tokyo University of Marine Science and Technology, Tokyo 108-8477, Japan \\ + Division of Navigation Science, Korea Maritime University, Busan 606-791, Republic. of Korea
}

\begin{abstract}
This study aims to identify the degree of safety when mariners take their actions in several different situations. We have carried out many experiments to observe mariners' behavior and then measured the safety level based on their actions to avoid dangerous situations of ships collision. One of the most important actions that mariners have to take, either as their daily routine or when they are in a collision situation and then want to avoid that situation is the lookout. In this paper, behaviors on the lookout have been defined as a standard sequence of three steps which are "time of first detection", "time of recognition as risky vessel" and "time of starting avoiding action", and the suitability and applicability of the definition have been shown. And also we propose the risk assessment on ships collision and the recommendation for reducing ships collision at sea. Some analyzing results and the application of the results are reported. By combining these knowledge and some systematic studies, we propose the risk assessment on ships collision and the recommendation for reducing ships collision at sea.
\end{abstract}

Key words : Degree of safety, Look-out, First detection, Recognition as risky vessel, Avoiding action, Risk assessment

\section{Introduction}

Safety degree of navigational situation is defined by the condition of navigational situation and mariner's competency that is realized at the given condition to maintain safe navigation(Park et al., 2008a; Iwanaga et al., 2005). In this paper, we discuss the safety degree on the collision avoidance maneuver by focusing on the mariner's standard behavior and the fluctuation(Kobayashi, 2009; Park et al., 2008b).

The important issues to decide the safety degree of collision avoidance maneuver are how to collect the necessary information and how to analyze them(Park et al., 2007a; Park et al., 2007b). The mariner's behavior concerning these processes is mainly achieved by the function of Lookout. Therefore we discuss the mariner's behavior on lookout in this paper.

First, we showed the standard mariner's behavior on lookout as standard performance. Secondly, we discussed the fluctuation of achievement degree of lookout caused by the environmental condition. Finally we proposed the assessment method on the navigational safety concerning collision avoidance maneuver based on the concept of necessary condition on safe navigation.

\section{Mariner's Standard Performance}

In this chapter, the main functions of lookout concerning collision avoidance will be explained. Data shown in this section are the experimental results conducted by the members of International Maritime Simulator Forum. Mariner's lookout is function of i) first detection, ii) recognition of risk of collision, and iii) starting avoiding action. Thus the mariner has the distinctive characteristics to each factors of the function.

\subsection{First Detection}

We examined the mariners' behaviour when they first detect the target vessel in crossing situation. Figure 1 shows a liner relationship between the distances to target vessel and the crossing angles with target vessel at the first detection (see Figure 2).

As the crossing angle becomes larger, the range of target vessels becomes bigger. The vessels with the larger crossing angle are nearly meeting in reciprocal courses each other. This result indicates the mariners may pay more attention to forward direction than aft when they are meeting.

\footnotetext{
* teddykim@hhu.ac.kr(051)410-4437

** kobayasi@kaiyodai.ac.jp

+ Corresponding author, songcu@hhu.ac.kr 051)410-4272
} 


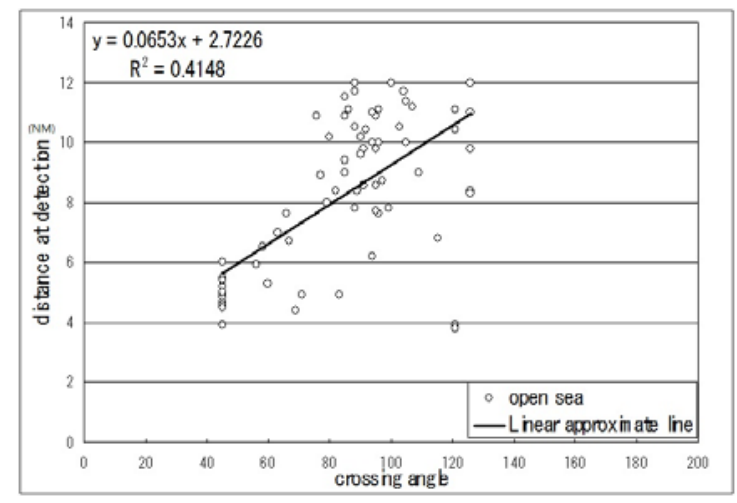

Fig. 1 The relation between the distances at first detection and crossing angles

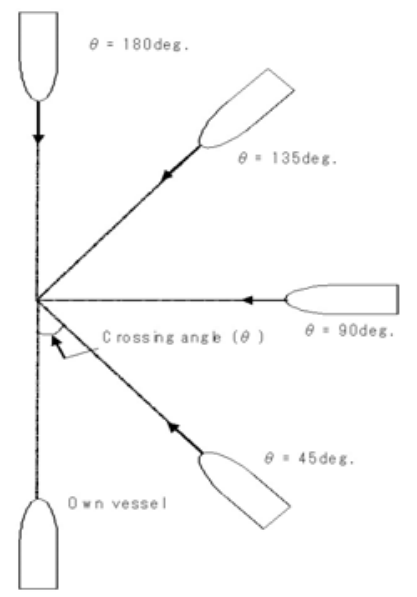

Fig. 2 The definition of crossing angle

We tested a linear relation between the crossing angle and the time to CPA (Closest Point of Approach) at the first detection. As shown in Figure 3, the data is scattered around the approximated linear line. It means the time to CPA at detection has almost no relation with the crossing angle from view point of residual time to collision. Mariners try to keep a specific time to CPA after detecting target. Mean time of first detection to CPA is about 25 minutes.

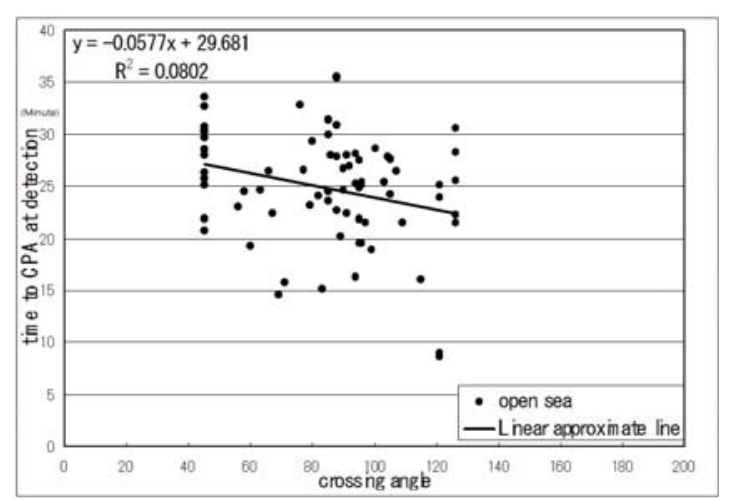

Fig. 3 The relation between the time to CPA at first detection and crossing angle

\subsection{Recognition as a risky vessel}

After the first detection, mariners continuously observe the target vessel to determine the collision risk. Figure 4 shows the time to CPA at the recognition of collision risk. The correlation between the crossing angel and the recognition time is weak, but there is the tendency where the recognition time of the risky vessels on forward direction becomes delayed.

The mean value of recognition time is 15 to 20 minutes. It means mariners judge the risks through 5 to 10 minutes observation after first detection.

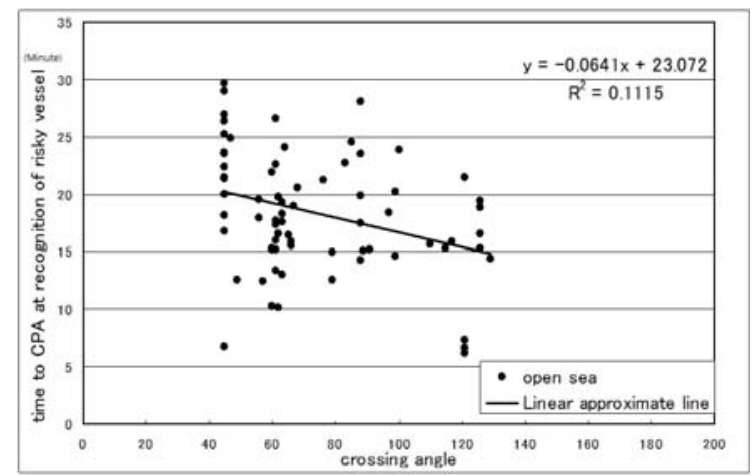

Fig. 4 The relation between the time to CPA at recognition and crossing angle

\subsection{Starting time of collision avoidance maneuver}

After recognizing the risk of collision, mariners keep observance of the target vessel, decide when to start avoiding action, and how to maneuver. Figure 5 shows the relation between the time at starting the collision avoidance action to CPA and the crossing angle.

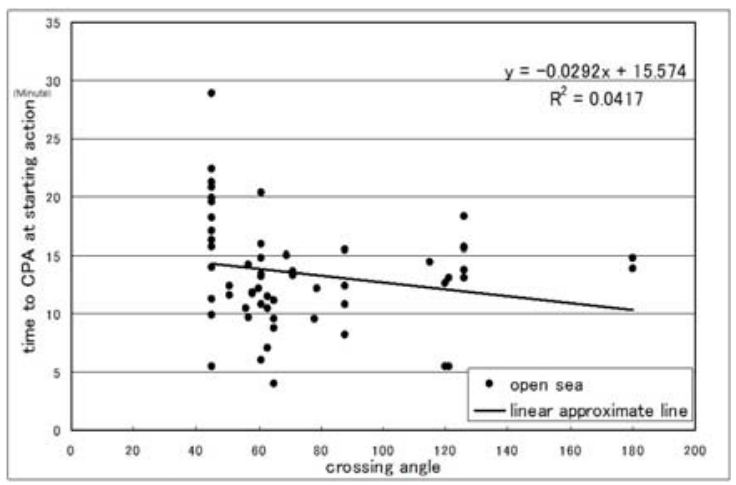

Fig. 5 The relation between the time to CPA at starting action and crossing angle

The correlation between the crossing angle and the starting time is not strong, but the tendency shows the starting time for avoidance on forward direction becomes 
delayed. The mean value of starting time is 10 to 15 minutes. It means mariners decide to start the avoidance action in 5 minutes after the time of risk recognition.

\section{Fluctuation of mariner's behavior}

\subsection{Change of detection caused by visibility}

Figure 6 shows the positive linear relation between the first detection and the visibility, which is expressed by radar range. In restricted visibility, mariners mainly detect the target vessel by observing RADAR/ARPA and changing its range scale, which depends on the visibility.

Generally, mariners use shorter range to detect the target vessel in restricted visibility than in clear visibility. Thus, in restricted visibility, they use the half range comparing with the range they normally use in clear visibility. The average time of the first detection is 25 minutes in clear visibility before the collision, so that it may reduce to half of the time in restricted visibility.

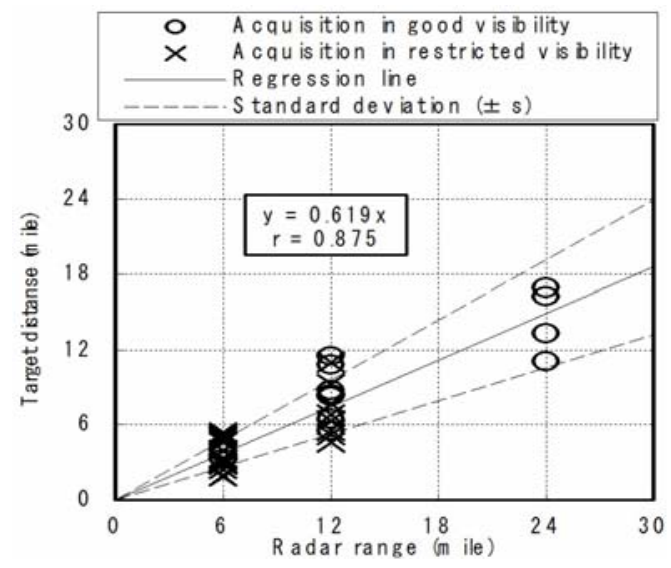

Fig. 6 The relation between the time of the first detection and visibility

\subsection{Change of the detection caused by traffic density}

The detection time is also changeable due to the workloads of mariners. They cannot keep sharp lookout when they have to achieve many tasks such as positioning, communication and so on. Especially, in case of heavy traffic condition, they have to pay attention to many vessels in the vicinity.

Usually, they pay more attention on the vessels nearby rather than those in further range. Thus they cannot achieve a sufficient level of observation on the further vessels, which causes the late detection. It means we can define the workloads as a positive function on the number of the vessels in the vicinity.

Figure 8 shows the relation between the time to CPA at first detection expressed by detection range (vertical axis) and the traffic density (horizontal axis). As shown in Figure 7 , traffic density is defined by the number of the vessels in the vicinity, and the vicinity area is defined by the circle with 3 miles of the diameter and own ship's position is 2 miles behind of the center of circle. Therefore we found that the workloads of lookout on the vessels in the vicinity have a negative effects on the detection range, which is equal to the first detection time.

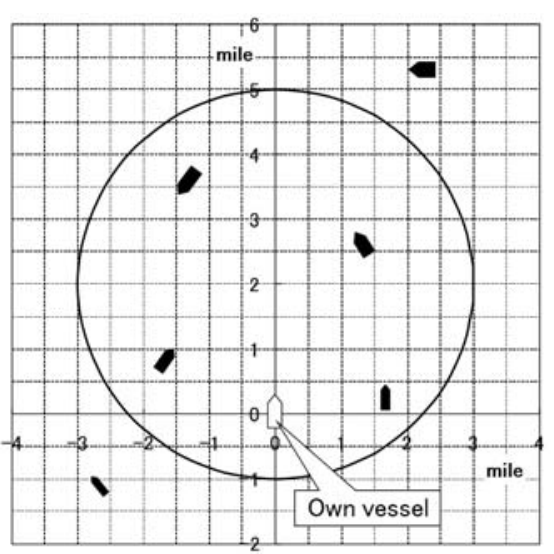

Fig. 7 Definition of traffic density in vicinity

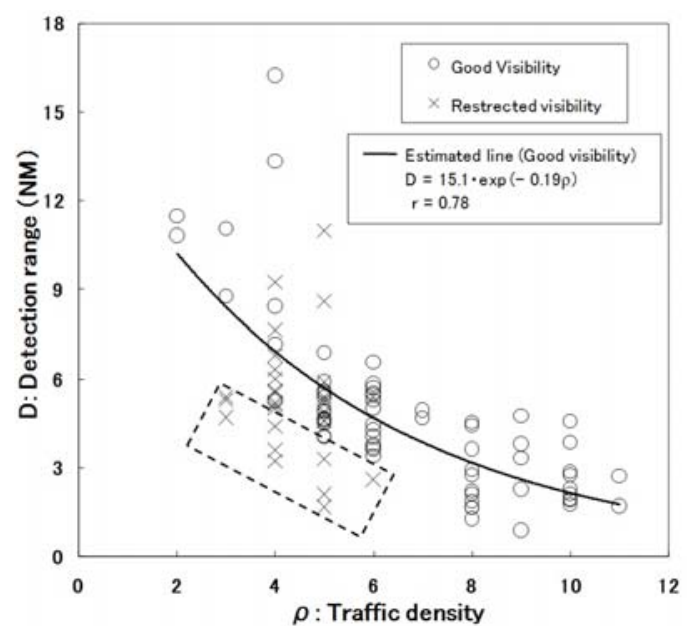

Fig. 8 First detection range shown by the relation to the traffic density

\subsection{Change of recognition time on collision risks by traffic density}

The change of recognition time due to the traffic density is shown in figure 9. It shows no changes to the traffic density. It is one of possibility that they pay attention to the target after they detected them and recognize the risk through continuous observation. 
The time to recognize the risks should be 15-20 minutes before collision. In case of heavy traffic condition over 4 ships in vicinity, mariners have to judge the risks of collision within 2 minutes after detection.

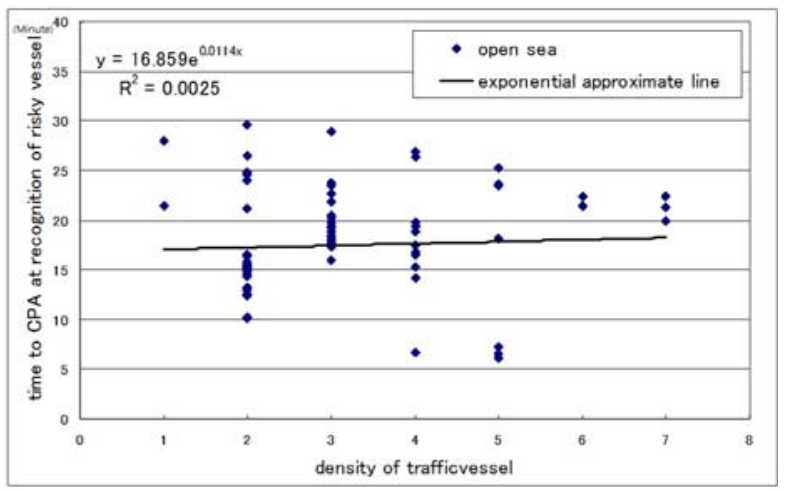

Fig. 9 The relation between the time to CPA at recognition and traffic density

\section{Human factor and navigational condition}

In this chapter, we explain necessary condition for safe navigation from viewpoint of the relation between mariner's function and the condition of navigational environment. This concept is very important to understand the necessity level of mariner's competency and the necessary condition of navigational environment for safe navigation.

We show that mariners having low competency and difficult navigational environments have higher potential to invite an accident. Furthermore, it is shown that mariners having high competency have also the possibility to cause an accident.

\subsection{Necessary condition of safe navigation}

Accidents are caused by many kinds of factors. Before discussing each factor in detail, it is necessary to discuss the condition of accidents occurrence from point of global view related with the factors on accidents. It is frequently mentioned that human factors contribute to the causes over $80 \%$ of accidents. On the other hand, it is also common understandings that the degree of safety level of navigation may be changed by the condition of navigational environments.

The concept on the condition is explained in this section based on the relationship between the difficult condition of navigational environments and human competency which decides the human behavior.

First, the conditions of navigational environment are discussed and listed as follows;

$\triangleright$ Maneuvering characteristics of own vessel

$\triangleright$ Water area for navigation

$\triangleright$ Weather and sea state

$\triangleright$ Traffic condition(kinds of traffic vessels and the density)

$\triangleright$ Requirement of traffic rule

Maneuverability, geographic condition and traffic rules are constant condition at the specific area, but the other factors mentioned above are not constant and changeable with uncertainty. The probability of difficulty is changeable with the condition of relating factors. They are the change of traffic density in term of time and the weather conditions and so on.

Secondly, the characteristics of human ability on ship handling are explained. The competency of human operator is mainly decided by following factors;

$\triangleright$ Mariner's license rank

$\triangleright$ Experiences

$\triangleright$ Fatigues(relating to the elapsed time of standing watch)

$\triangleright$ Tension(relating to the time of watch)

Human does not always show the constant competency. In general, it is changing with time. The factors that make the change of competency are fatigue and tension. In case of high tension, he or she shows higher competency than normal competency. Therefore, the safety level of navigation is decided by the factors mentioned above.

\subsection{Occurrences of accident and its condition}

In this section, the relationship between the accident occurrence and its conditions is explained. The risks of handling ship should not only be determined by human competency, but it is determined by the condition of navigation environment.

The conditions of accident occurrence relate to the combination between the condition of navigational environments and the human competency. Figure 10 shows the relation between the competency required by environment to accomplish safe navigation at the condition and the human competency.

The line with an incline of 45 degrees indicates the equivalent condition between them. Normal navigation is carried out in the condition of upper part of this line. They are safe situation. In the lower zone of this line, it shows a dangerous situation as occurrence of accident. This area shows that the competency required by the navigational environment needs higher than mariners' competency. 
Figure 11 shows the situation of Figure 10 with the fluctuation of both factors. In case of the condition with both competencies being average shown as situation "A", the safe navigation can be expected. As mentioned above, the condition of human and environment may not be constant and they may fluctuate.

With the change of weather and the concentration of traffic vessels, the required competency becomes temporarily high. This condition is shown as situation "B". Safe navigation cannot be accomplished by the average human competency at this situation. If human realizes higher competency with tension and so on, the situation can be moved to the situation " $\mathrm{C}$ ", and then becomes safe again.

On the other hand, although the navigation environment is the mean situation, human competency shows lower one by the fatigue or lack of tension. This situation shown by " $\mathrm{D}$ " is also dangerous situation.

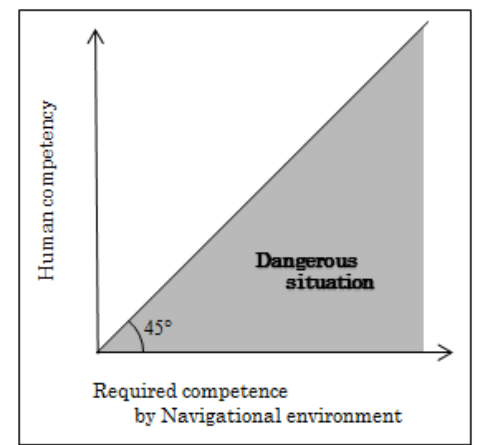

Fig. 10 The navigational safety defined by both condition of human competency and required competency by navigational environment

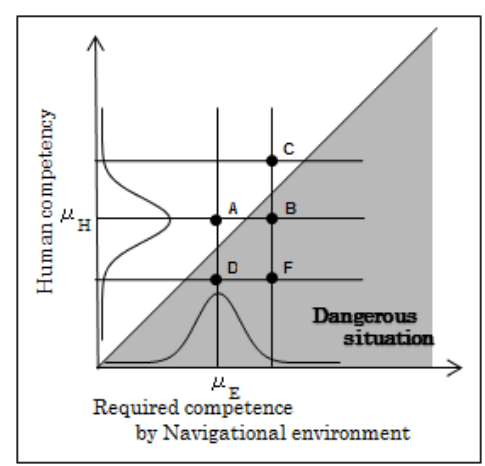

Fig. 11 The change of safety degree relating to the probability of human competency and required competency

By analyzing causes of marine accidents, it is difficult to recognize the necessary conditions which lead to the accidents. In other words, although the similar situation often happens daily, it is possible that no accident occurs, so it is reasonable that the accident may happen when the unusual events occur simultaneously. It is statistic event.

One of the situations that rarely happen is shown by situation " $\mathrm{F}$ " in Figure 11. In this condition, the ability required by the navigational environment becomes extremely high while the human ability goes down temporary.

\subsection{Navigation safety decided by Mariner's performance}

The degree of safe navigation is decided as mentioned in previous chapter. When the navigational condition shows specific situation, safety degree is decided by mariner's performance. The experienced mariners show the standard performance shown in section 1 . When the difficulty of navigational condition is higher than standard mariner's competency, the maritime accidents may occur.

However, standard mariner's performances shown in chapter 2 are related to mariners' behaviors in the specific navigational condition, and those behaviors are changeable due to the condition shown in chapter 3. Therefore, when we discuss the safety degree of navigation, we have to take the fluctuation of mariner's performance into account.

The fluctuations of mariner's behavior shown here are caused by the change of the navigational condition but the fluctuations appear caused by mariner's situation. When mariner becomes tired, mariner shows lower awareness and may execute delayed detection. The fluctuation deviating from standard performance is also shown caused by the different mariner's competency. Thus, we have to study about the fluctuation of mariner's competency when we estimate the safety degree of navigation in actual maritime activities.

\section{Conclusion}

Safe navigation is a top priority in maritime activities. However, when an accident occurs, the discussion on the causes are always executed to analyze the lack of mariner's performance. The action right before the accident is done by human in most of cases.

Then the causes of accidents are assessed as the incorrect mariners' behavior. In other cases, the causes are estimated as an insufficient function that should be conducted by mariner. In case of collision, causes are estimated as the delayed detection and/or insufficient continuous lookout to estimate the situation. 
However, in case of hard navigational situation, normal mariner cannot achieve the standard mariner's function and then he cannot has sufficient information and carry out proper handling. We have to consider what maximum mariner's competency is and what necessary condition is to maintain safe navigation. Necessary discussion to achieve safe navigation will be dealt with in the future study.

\section{References}

[1] Kobayashi(2009), "Mariner's Function for Safe Navigation", MARSIM Conference.

[2] Park, J.S., Kobayashi, H. and Yea, B.D.(2008a), “A Study on Mariners' Standard Behavior for Collision Avoidance(3)", Journal of Navigation and Port Research International Edition, Vol.32, No.4, pp. 279 285.

[3] Park, J.S. and Yea, B.D.(2008b), “A Study on the Influence of Navigation Environment on Mariner's Behavior for Collision Avoidance”, Journal of Navigation and Port Research International Edition, Vol.32, No.2, pp. 127 132 .

[4] Park, J.S., Kobayashi, H. and Yea, B.D.(2007a), “A Study on Mariners' Standard Behavior for Collision Avoidance(2)", International Journal of Navigation and Port Research, Vol.31, No.4, pp. 309 315.

[5] Park, J.S., Kobayashi, H. and Yea, B.D.(2007b), “A Study on Mariners' Standard Behavior for Collision Avoidance(1)”, International Journal of Navigation and Port Research, Vol.31, No.4, pp. 281 287.

[6] Iwanaga, K. and Kobayashi, H.(2005), "A Study on the relation between the difficulty of avoiding collision and mariner's behaviors", Proceeding of $5^{\text {th }}$ Asian Conference on Marine Simulator and Simulation Research, pp. 71 77.

Received 15 May 2012

Revised 27 June 2012

Accepted 27 June 2012 